\section{DEVELOPMENT OF A NATIONAL EMS POLICY FOR KENYA: OPPORTUNITIES FOR ACTION}

${ }^{1}$ Isaac M Botchey, ${ }^{1}$ Fatima Paruk, ${ }^{2}$ Daniel Wako, ${ }^{3}$ Wilson Gachari, ${ }^{3}$ Simon Kibias, ${ }^{1}$ Adnan A Hyder, ${ }^{1,4}$ Kent A Stevens. ${ }^{1} J o h n s$ Hopkins International Injury Research Unit, USA; ${ }^{2}$ Centresfor Disease Control and Prevention, Kenya; ${ }^{3}$ Ministry of Health, Kenya; ${ }^{4}$ Johns Hopkins Hospital Department of Surgery, USA

\subsection{6/injuryprev-2016-042156.785}

Background Emergency Medical Services (EMS) are a community's gateway to acute and emergency medical care for members of the public facing time-sensitive, critical illness and injury. ${ }^{1}$ A functional EMS is an effective, frontline intervention to reduce the disproportionately high morbidity and mortality in Low- and-Middle Income Countries (LMICs). ${ }^{1-3}$ The World Health Organisation and the African Federation for Emergency Medicine have promoted the formation of locally appropriate EMS systems in LMICs. ${ }^{1-4}$

Description of the problem Under article 43 of the Constitution of Kenya "a person shall not be denied emergency medical treatment." However, recent events including floods and the Westgate terrorism attack have revealed a low-functioning system for care of the injured in Kenya. There is extensive variability in the level of care provided at the pre-hospital setting due to the absence of national standards in training of personnel, available equipment and infrastructure. Furthermore, emergency rooms in the public health facilities are often poorly resourced to deliver definitive emergency care.

Results In 2012 and 2013, Johns Hopkins International Injury Research Unit, CDC-Kenya and the Ministry of Health brought together local EMS stakeholders to form a consortium. Members were tasked with creating a locally appropriate EMS Policy. In 2015, a comprehensive policy that recognises pre-hospital care as a component of the healthcare system was developed. It establishes a regulatory body for EMS, defines minimum training and equipment standards and mandates data reporting for quality improvement.

Conclusions The development of a locally appropriate EMS policy requires consensus and extensive stakeholder engagement. The implementation of this policy will provide the opportunity for definitive emergency care as stipulated in the national constitution and serve as a model for EMS development in LMICs.

\section{REFERENCES}

1 Kobusingye $O C$ et al. Emergency medical systems in Low-and middle-income countries: recommendations for action. Bulletin of the World Health Organization 2005;83:626-31

2 Razzak JA and Kellermann AL. Emergency medical care in developing countries: is it worthwhile? Bulletin of the World Health Organization 2002;80:900-905

3 Henry JA and Reingold AL. Prehospital trauma systems reduce mortality in developing countries: a systematic review and meta-analysis. Journal of trauma and Acute Care Surgery 2012;73:261-268

4 Calvello $\mathrm{E}$ et al. Emergency care in sub-Saharan Africa: Results of a consensus conference. African Journal of Emergency Medicine 2013;3:42-8

\section{IN-HOSPITAL MORTALITY BASED ON THE MODE OF EMS TRANSPORTATION IN HIGH-INCOME DEVELOPING MIDDLE EASTERN COUNTRY}

${ }^{1}$ Hassan Al-Thani ${ }^{1}$ Ayman El-Menyar ${ }^{2}$ Yugan Pillay, ${ }^{1}$ Monira Mollazehi ${ }^{1}$ Ahammad Mekkodathil, 'Rafael Consunji. 'Trauma Surgery; ${ }^{2}$ EMS Services, Hamad General Hospital, Doha, Qatar

10.1136/injuryprev-2016-042156.786

Background Helicopter emergency medical services (HEMS) have become a standard element of advanced pre-hospital care system. Positive outcomes in HEMS transportation were evident in different settings and certain types of patients. The present study assessed presentations and outcomes in trauma patients transported by HEMS and ground emergency medical services (GEMS) in Qatar.

Methods A retrospective review of trauma registry data at Hamad Trauma Centre (HTC) was conducted for trauma patients transported by GEMS and HEMS in the duration from 2011 to 2013. Patients were classified into two groups based on the mode of transportation (HEMS vs GEMS). Injury severity score (ISS) was defined as severe/profound trauma $(>15)$ and mild/moderate trauma $(\leq 15)$. Mortality was defined as brought in dead (BID), within the first day, within the first week and more than 1 week.. Results A total of 4596 patients with traumatic injuries were included (7\% HEMS). When compared with GEMS, the mean ISS, Head AIS and scene GCS were greater in HEMS ( $p=0.001$ ). Nearly $12 \%$ of ISS $>15$ and $5 \%$ of ISS $\leq 15$ were transported by HEMS. The main mechanism of injury was motor vehicle crash (MVC) (37\%) followed by fall (25\%) and pedestrians (14\%). MVC victims were more likely to be transported by HEMS whereas fall and pedestrians victims were frequently transported by GEMS ( $p<0.05$ ). On-scene rate of intubation was higher in HEMS irrespective of ISS score $(p=0.001)$. Overall mortality, in-hospital deaths and BID was 10.2\%, 7.5\% and $3 \%$; respectively. The mortality was 2.5 -fold higher in victims transported by HEMS compared with GEMS (10\% vs 4\%, $\mathrm{p}=0.001)$. Mortality within the first day and more than one week were higher with HEMS, however, it was greater within the first week in GEMS. Age and mode of transportation adjusted predictors of mortality were ISS and scene GCS.

Conclusions MVCs are the main mechanism of injury in Qatar. Polytrauma and scene GCS play important prognostic role regardless the mode of transportation. These findings indicate the need of more effective preventive measure on the road

\section{THE EEFECT OF PREHOSPITAL INTRAVENEOUS FLUID ON SURVIVAL OF TRAUMA PATIENTS IN KHON KAEN HOSPITAL}

Porntipa Tantibundit, Somporn Hongveang. Emergency Department Khon Kaen Hospital, Thailand

\subsection{6/injuryprev-2016-042156.787}

Background The administration of intravenous fluid (IV fluid) during prehospital setting for trauma patients is the traditional procedure in emergency medical service (EMS) system. We believed that intravenous fluid would maintain intravascular volume and organ perfusion. Conversely, the prehospital IV fluid access procedure increased the on scene time and missed the concept scoop and run for definite treatment and in one theory IV fluid was cause of rebleeding from elevation of blood pressure. This study aimed to identify the effect of prehospital IV fluid administration on survival to discharge of trauma patients in Khon Kaen hospital.

Methods This was retrospective cohort study. Trauma patients who arrived by EMS system and admited in Khon Kaen hospital during $1^{\text {st }}$ January $2014-31^{\text {th }}$ December 2014 were enrolled. The effect of prehospital IV fluid and other important variables on survival to discharge were analysed by multiple logistic regression.

Results There were 2,610 patients who arrived by EMS system and all of them were admited. $4.4 \%$ of patients received IV fluid from prehospital setting. Survival was higher in patients who not 